\title{
A central role for MeCP2 in the epigenetic repression of miR-200c during epithelial-to- mesenchymal transition of glioma
}

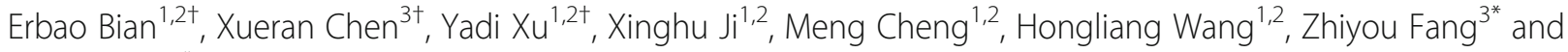 \\ Bing Zhao ${ }^{1,2^{*}}$
}

\begin{abstract}
Background: The epithelial-to-mesenchymal transition (EMT) has been linked to the regulation of glioma progression. However, the underlying signaling mechanisms that regulate EMT are poorly understood.

Methods: Quantitative real-time PCR (RT-qPCR) and western blot were performed to detect the expression of MeCP2 in glioma tissues and cell lines. MeCP2 functions were tested with cell immunofluorescence staining and western blot. For in vivo experiments, mouse xenograft model was used to investigate the effects of MeCP2 on glioma. ChIP and Co-IP were used to detect the relationships among MeCP2, miR-200c and Suv39H1.

Results: In this study, we found that MeCP2 was frequently up-regulated in human glioma tissues and cell lines. MeCP2 knockdown remarkably induced cell epithelial phenotype and inhibited mesenchymal marker ZEB1 and ZEB2 in vitro and in vivo. In addition, MeCP2 in glioma tissues was negatively correlated with miR-200c expression, and miR200c overexpression partially abrogated mesenchymal phenotype induced by MeCP2. More importantly, we showed that MeCP2 recruited H3K9 to the promoter of miR-200c by interacting with SUV39H1, resulting in EMT of glioma cells.

Conclusions: This study for the first time reveals MeCP2 as a novel regulator of EMT in glioma and suggest that MeCP2 inhibition may represent a promising therapeutic option for suppressing EMT in glioma.
\end{abstract}

Keywords: Glioma, MeCP2, miR-200c, SUV39H1

\section{Background}

Gliomas are the most common primary brain tumor characterized by highly infiltrative growth. Base on the pathological characteristics, gliomas can be classified into four clinical grades. (Glioblastoma multiforme, GBM) is one of the most aggressive types of brain tumors, and despite the combination of multiple treatments, including surgery, chemotherapy and radiation, patients often still develop refractory recurrence [1]. In general, GBM patients have a median survival time of no more than 16

\footnotetext{
* Correspondence: z.fang@cmpt.ac.cn; aydzhb@126.com

†Erbao Bian, Xueran Chen and Yadi Xu contributed equally to this work.

${ }^{3}$ Anhui Province Key Laboratory of Medical Physics and Technology, Center of Medical Physics and Technology, Hefei Institutes of Physical Science, Chinese Academy of Sciences, No. 350, Shushan Hu Road, Hefei 230031, Anhui, China

'Department of Neurosurgery, The Second Affiliated Hospital of Anhui Medical University, 678 Fu Rong Road, Hefei 230601, Anhui Province, China Full list of author information is available at the end of the article
}

months after optimal treatment [2]. GBM are classified into four molecular subtypes including mesenchymal, classical, proneural and neural subtypes based on gene expression-based molecular classification [3].The mesenchymal GBM subtype has recently been shown to be the most malignant with resistance to radiotherapy and chemotherapy. This pathogenic phenotype has been associated with the epithelial-to-mesenchymal transition (EMT). The EMT is a key biological process that is normally involved in embryonic development and have been reported to regulate invasion and metastasis of tumor $[4,5]$. In GBM, members of the ZEB-family, e.g., ZEB1 and ZEB2, known as the activators of EMT, can promote the invasiveness of GBM cells [6, 7]. Therefore, understanding the molecular mechanism of EMT is essential for the development of novel and effective therapeutic strategies for gliomas.

(c) The Author(s). 2019 Open Access This article is distributed under the terms of the Creative Commons Attribution 4.0 International License (http://creativecommons.org/licenses/by/4.0/), which permits unrestricted use, distribution, and reproduction in any medium, provided you give appropriate credit to the original author(s) and the source, provide a link to the Creative Commons license, and indicate if changes were made. The Creative Commons Public Domain Dedication waiver (http://creativecommons.org/publicdomain/zero/1.0/) applies to the data made available in this article, unless otherwise stated. 
Methyl CpG-binding protein $2(\mathrm{MeCP} 2)$ is a member of the methyl-CpG-binding domain (MBD) family of proteins [8]. MeCP2 has been found to have two functional domains, a 104-amino-acid transcriptional repression domain (TRD) and an 85-amino-acid MBD. MBD binds DNA sequences methylated at cytosine in the dinucleotide $5^{\prime}$-CpG and TRD acts as a transcriptional repressor by recruiting histone deacetylase complex (HDAC) $[9,10]$. $\mathrm{MeCP} 2$ has been reported to be implicated in a number of molecular functions, such as transcription regulation, RNA splicing, and chromatin organization [11, 12]. Lossof-function mutations in MeCP2 causes Rett syndrome (RTT), whereas the duplications of MeCP2-containing loci may result in spectrum of phenotypes ranging from autism to intellectual disabilities and mood disorders $[13,14]$. Recently, it has been reported that a non-neuronal role for $\mathrm{MeCP} 2$ has emerged in tumorigenesis, such as prostate cancer, breast cancer and gastric cancer $[15,16]$. However, little is known about its biological characteristics and molecular mechanisms in human glioma.

MicroRNAs, known as small noncoding RNAs, are involved in regulation of downstream gene expression at the posttranscriptional level [17]. Most importantly, the complex regulatory network not only allows one gene by the combination of multiple microRNAs, but also modulates the expression of several genes via one microRNA $[18,19]$. Recently, numerous deregulated miRNAs have been reported to be associated with human cancers progression $[20,21]$. The miR-200 family (miR-200c/ miR-141 and miR-200a/miR-200b/miR-429 clusters) is a tumor-suppressive group of miRNAs that is implicated in suppressing EMT process [22, 23]. Recent reports have suggested that the repression of miR-200 s expression occurs due to epigenetic modification, such as DNA methylation and histone methylation [24-26]. In addition, our previous study showed that miR-141 methylation mediated by DNMT1 in glioma. However, the association of MeCP2 with miR-200s dysregulation is still unknown.

In this paper, we found that $\mathrm{MeCP} 2$ knockdown repressed EMT process of glioma cells, and expression of ZEB1 and ZEB2. Additionally, MeCP2 regulated the expression of microRNA-200 family targeting ZEB1 and ZEB2 transcripts through epigenetic modification. The present work reveals epigenetic regulatory network between miRNA and methylation, which will provide a novel therapy strategy for guarding against EMT of gliomas via targeting $\mathrm{MeCP} 2$ and its downstream miR-200c.

\section{Materials and methods}

\section{Patients and tissue samples}

Resected brain tumors were collected from the Department of Neurosurgery of The Second Affiliated
Hospital of Anhui Medical University (Hefei, China) after obtaining all patient's or their client's informed consent. Tissue samples included 12 normal brain tissues and 65 glioma tissues. All cases were pathologically graded as low grade (WHO I/II, $n=22$ ) and high grade (WHO III/IV, $n=43$ ) according to the WHO criteria, and the information about the essential characteristics of these tumors was placed in Additional file 1: Table S1. Samples were preserved in liquid nitrogen and stored in $-80^{\circ} \mathrm{C}$. This experiment was approved by the Research Ethics Committee of The Second Affiliated Hospital of Anhui Medical University.

\section{TCGA data assay}

The Cancer Genome Atlas (TCGA) assay was carried out by website http://cancergenome.nih.gov/ as previous described [27]. This TCGA cohort include normal tissues $(n=207)$, low grade $(n=518)$ including all lowgrade gliomas, and GBM $(n=163)$, and genes expression were analyzed. In addition, GBM subtypes expression data according to their molecular classification (classic, proneural, neural, mesenchymal) were obtained and the relationship between the expression of genes in glioma was analyzed by in this database.

\section{Cell culture}

Glioma cell lines (U251, LN18, A172, and U87) and normal human astrocytes (NHAs) were obtained from the Cell Bank of the Chinese Academy of Sciences (Shanghai, China) and Sun Yat-Sen University, respectively. Cells were grown in DMEM and supplemented with $10 \%$ fetal bovine serum (FBS, Gibco, USA), $100 \mathrm{U} /$ $\mathrm{ml}$ penicillin/streptomycin (Sigma), in the presence of $5 \% \mathrm{CO}_{2}$ at $37^{\circ} \mathrm{C}$.

\section{Immunofluorescence staining}

Cells were inoculated into the 6-well plate comprising slide and cultured overnight. After that, cells were fixed with 4\% paraformaldehyde (Beyotime Biotechnology, ST476). Cells were permeated with $0.5 \%$ Triton X-100 (Beyotime Biotechnology, ST795) in PBS and blocked with $5 \%$ bovine serum albumin (BOSTER, AR0004) and incubated with $\beta$-tubulin (cell signaling,cat\#2146), ZEB1 (Abcam, ab245283) and ZEB2 (Abcam,ab138222) antibody overnight at $4{ }^{\circ} \mathrm{C}$.FITC conjugated secondary antibody was used to detect primary antibody, and then DAPI was used to incubate cells for counterstaining. Images were acquired using a DP71 fluorescence microscope with a digital color camera (Olympus, USA).

\section{Cell transfection}

The human MeCP2-WT gene and other constructs of $\mathrm{MeCP} 2$ truncations $\mathrm{MeCP} 2^{\Delta \mathrm{N}}$, and $\mathrm{MeCP} 2^{\Delta \mathrm{C}}$ were generated according to manufacturer's instructions. Sh- 
MeCP2 (5' -TGCTTAAGCAAAGGAAATCTCTCGAG AGATTTCCTTTGCTTAAGCTTTTTTC-3') was provided by GenePharma (Shanghai, China), and its corresponding non-targeting sequence (sh-control). si-SUV39H1 and nonspecific control siRNA (si-NC) were synthesized (Genepharma, Shanghai, China). Plasmid was transfected into U251 and LN18 glioma cells respectively by using Lipofectamine 2000 (Invitrogen, USA) according to the manufacturer's protocol. MiR-200c mimics and miR-200c negative control (NC) were purchased from RiboBio (Guangzhou, China), and transfected into cell lines according to the manufacturer's protocol.

\section{RNA extraction and quantitative real-time PCR}

Total RNAs from tissues and cultured cells were extracted using Trizo reagent (Invitrogen, USA) according to the manufacturer's protocol. cDNA was reversely transcribed by using PrimeScriptTM RT Master Mix (Perfect Real Time) (TaKaRa Biotechnology, China). Allin-One $^{\mathrm{Tw}}$ miRNA First-Strand cDNA Synthesis Kit (Genecopoeia, China) was used for miRNA reverse transcription and RT-qPCR was performed using All-in$\mathrm{One}^{\mathrm{rn}}$ miRNA qPCR Kit (Genecopoeia, Guangzhou, China) of miR-200a (Cat\#HmiRQP0298), miR-200b (Cat\#HmiRQP0300), miR-200c (Cat\#HmiRQP0302), miR141(Cat\#HmiRQP0184), miR-429 (Cat\#HmiRQP0497) and U6 (Cat\#HmiRQP9001). Real-time PCR was performed with SYBR Green detection chemistry (TaKaRa Biotechnology, China) on ABI 7500 Real-Time PCR System (Applied Biosystems). For relative quantification, $2^{-\Delta \Delta C t}$ was calculated, with U6 RNA as a reference in miRNA analysis and GAPDH as a reference in the analysis of protein coding genes. The indicated primers were placed in Additional file 2: Table S2.

\section{Western blotting}

Tissues and cells were treated with RIPA lysis buffer (Beyotime, China). Total protein extracts (20 or $40 \mu \mathrm{g}$ ) were subjected to $8 \%$ or $12 \%$ SDS PAGE separation and then transferred to PVDF membrane (MilliporeCorp, U.S.A.). Membranes were probed with targeted primary antibodies: Anti- $\beta$-actin (Abcam, ab179467), Anti-MeCP2 (Abcam, ab195393), anti-ZEB1 (Abcam, ab203829) and anti-ZEB2 (Abcam, ab138222). Following blots were washed three times in TBS/Tween-20 and then incubated with appropriate horseradish peroxidase (HRP)-conjugated secondary antibody at a 1:10000 dilution in TBS/ Tween-20 containing 5\% milk. Proteins were visualized using an ECL chemiluminescence kit (ECL-plus, Thermo Scientific).

\section{Co-immunoprecipitation (co-IP) assay}

Co-IP assays were performed to examine the interaction between MeCP2 and SUV39H1 according to the manufacturer's protocol, and complexes were precipitated with protein A/G agarose (Bimake, B23201). Then, complexes were subjected to western blot analysis using anti-MeCP2 (Abcam, ab195393), anti-SUV39H1 (Abcam, ab12405).

\section{Chromatin immunoprecipitation (ChIP)}

ChIP assays were performed according to previously described Protocols [28]. In brief, ChIP was performed using the EZ-Magna ChIP Chromatin Immunoprecipitation Kit (Millipore). The antibodies were obtained from Abcam: anti-H3K9 (ab8898) and anti-SUV39H1 (Abcam, ab12405). The DNA was detected through RT-qPCR and primers were provided in Additional file 2: Table S2.

\section{Tumor formation study in vivo}

All mouse experiments were approved by the Animal Research Committee of Anhui Medical University. LN18 glioma cells infected with lentiviral vectors (GeneChemCo.Ltd., Shanghai, China) containing sh-MeCP2 and sh-con were suspended in $200 \mu \mathrm{PBS}$ and subcutaneously injected in $\mathrm{BALB} / \mathrm{c}$ female nude mice $(5 \times$ $10^{6}$ cells/mouse).Tumor size was measured every 7 days using an electronic caliper, and the tumor volume was determined with the formula: $\mathrm{V}=0.5 \times \mathrm{L}$ (length) $\times \mathrm{W}^{2}$ (width) [29]. Mice were sacrificed after 6 weeks at cell inoculation, and tumors were excised and evaluated for volume.

\section{Immunohistochemistry}

Samples were fixed in $4 \%$ paraformaldehyde, and then were dehydrated, embedded in paraffin, and sectioned. Immunohistochemistry staining was performed according to the manufacture's protocol using antibodies against ZEB1 and ZEB2 (Abcam, USA). Images were visualized using florescence microscope. Results of immunohistochemical (IHC) staining was analyzed by Image proPlus6.0.

\section{Statistical analysis}

Data are presented as mean \pm SD of three independent experiments, in which each assay was performed in triplicate. Statistical analysis was performed using the GraphPad Prism 5 software. The correlation between the expression of MeCP2, miR-200c, ZEB1 and ZEB2 in tissues was analyzed with Pearson's correlation. A $p$ value $<0.05$ was considered statistically significant.

\section{Results}

Increased MeCP2 levels in gliomas tissues and cells

To evaluate MeCP2 expression in glioma samples and cell lines, MeCP2 expression were detected by RTqPCR. As shown in Fig. 1a, MeCP2 mRNA expression 


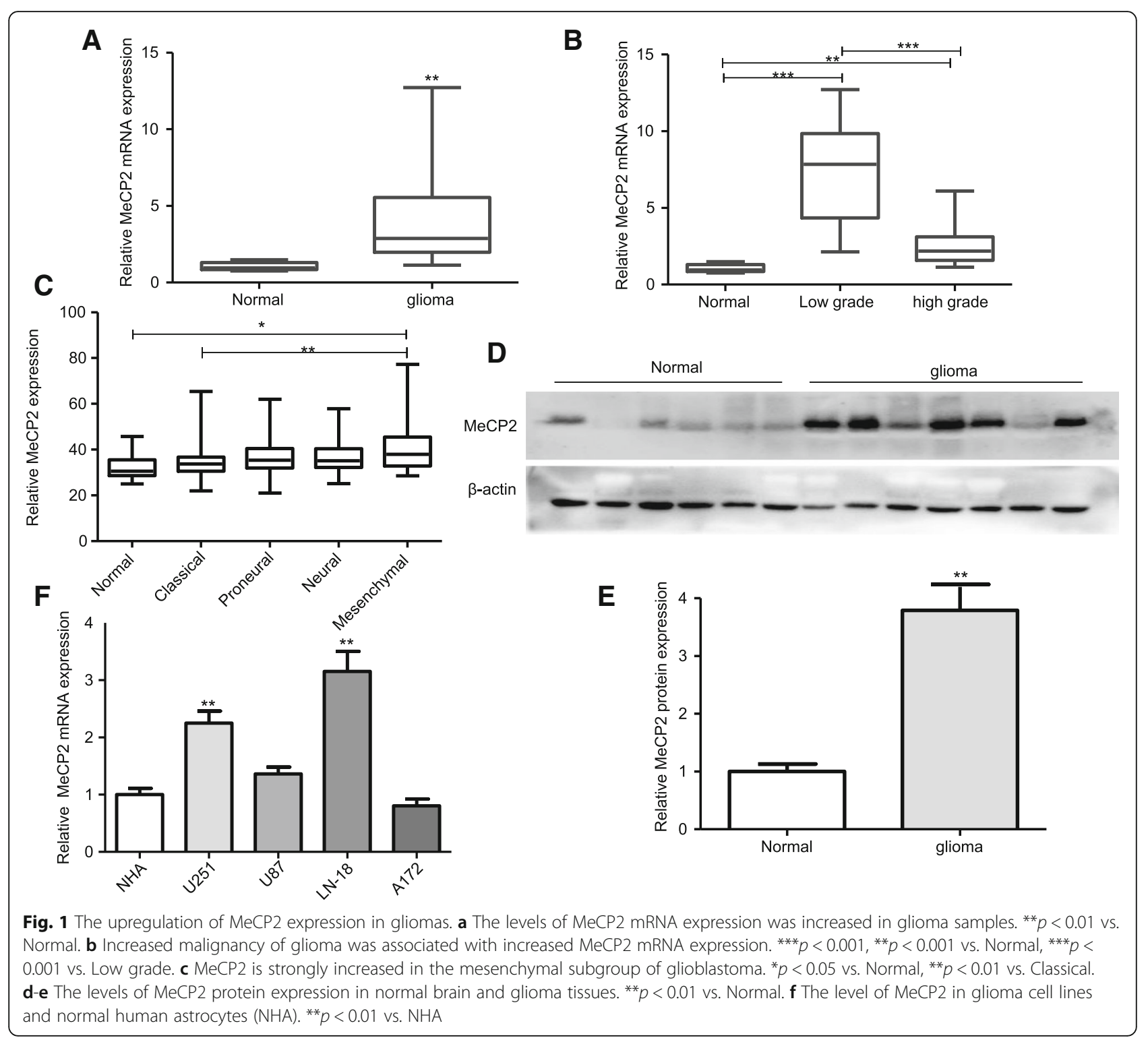

was markedly increased in glioma tissues compared with normal brain tissues. To determine whether the expression of MeCP2 is linked to the pathogenesis of glioma, MeCP2 expression in glioma tissues with different histopathologic grades was observed. A significant increase was observed in low grade glioma samples, whereas a slight increase in high grade glioma samples (Fig. 1b). Interestingly, MeCP2 expression is significantly increased in mesenchymal glioblastoma as compared with classical subgroup as divided after Verhaak et al. [3] (Fig. 1c). To confirm the changes of $\mathrm{MeCP} 2$ expression in gliomas, immunoblotting analysis was performed. MeCP2 protein expression was increased in glioma tissues compared with that in normal brain tissues (Fig. 1d-e). Moreover, we also measured
MeCP2 levels in a panel of glioma cell lines and a normal human astrocytes (NHA). Compared with NHA, MeCP2 expression levels were significantly increased in LN-18 and U251 glioma cell lines (Fig. 1f). Therefore, we chose LN-18 and U251 glioma cells for further functional studies.

\section{MeCP2 knockdown induces epithelial phenotype in vitro}

To investigate the effect of MeCP2 on EMT in gliomas, LN-18 and U251 glioma cells were transfected sh$\mathrm{MeCP} 2$ and sh-con. Decreased expression of MeCP2 after transfection of sh-MeCP2 was confirmed in LN-18 and U251 glioma cell lines (Fig. 2a-c). In addition, obvious cell morphology change was observed in the second weeks after MeCP2 knockdown, and cells morphology 


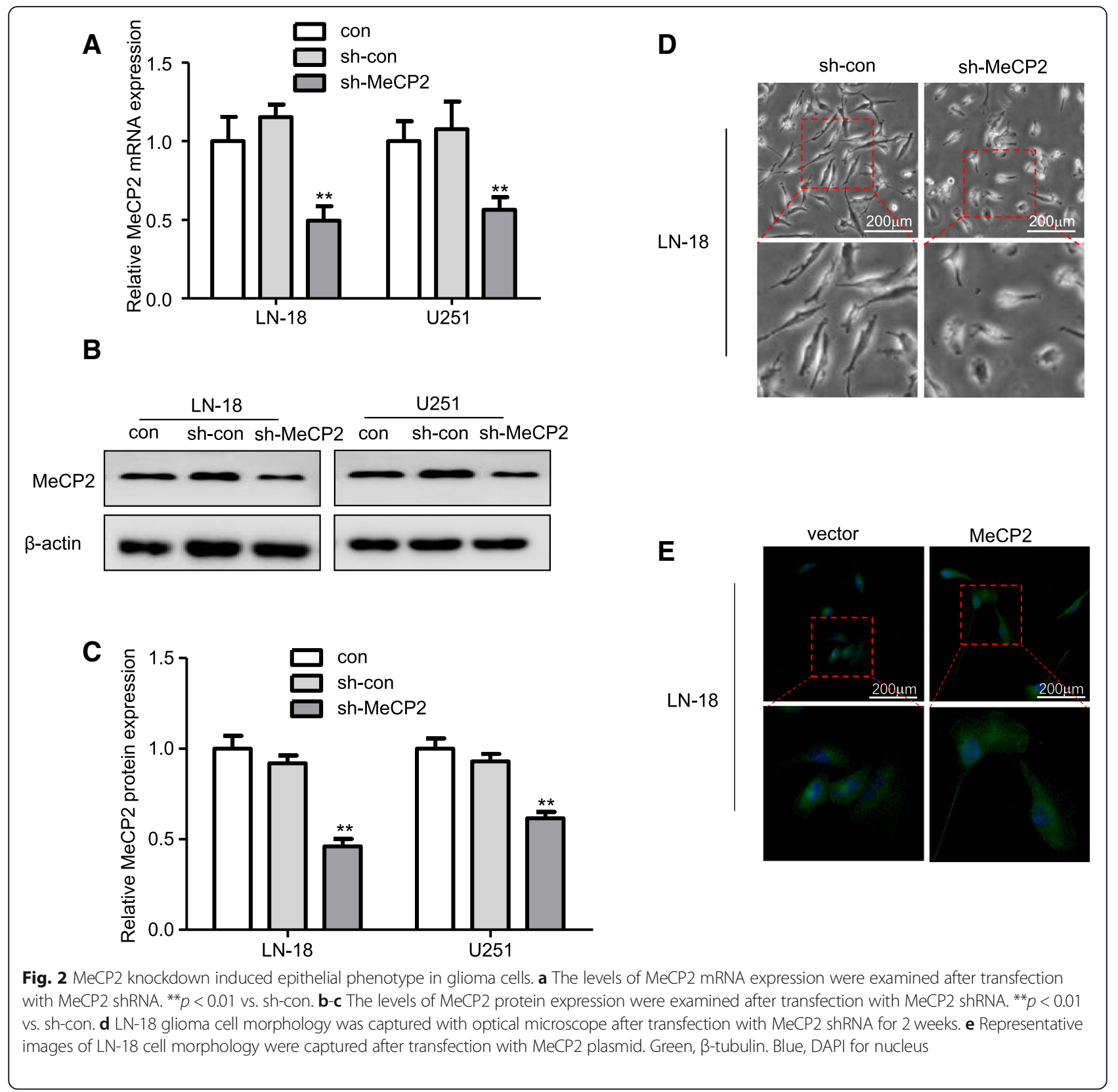

were changed from mesenchymal morphology to epithelial morphology (Fig. 2d and Additional file 3: Fig. S1a). Staining microtubules by $\beta$-tubulin antibody was used to detect the morphological changes. As shown in Fig. 2e and Additional file 3: Fig. S1b-c, MeCP2 overexpression increased spindle shaped morphology compared with vector. To determine whether MeCP2 regulate EMT in glioma, the expression of EMT markers was analyzed. We found that knockdown of MeCP2 increased mRNA expression levels of epithelial markers E-Cadherin, and decreased mesenchymal markers, ZEB1, ZEB2, and TWIST1 in LN18 and U251 glioma cells (Fig. 3a-b).
Because MeCP2 could upregulate ZEB1, ZEB2, TWIST1 and E-Cadherin, we next examined whether MeCP2 is coexpressed with ZEB1, ZEB2, TWIST1 and E-Cadherin in human glioma samples. TCGA database analysis showed that ZEB1, ZEB2 and TWIST1 expression (Fig. 3c-e), but not E-Cadherin (Additional file 3: Fig. S1d), were increased in glioma. As shown in Fig. 3fg, expression of ZEB1 and ZEB2 was significantly increased in glioma tissues compared with normal tissues. In addition, MeCP2 expression was positively correlated with ZEB1 and ZEB2 expression in the TCGA cohort (Fig. 4a-b), but not correlated with TWIST1 and 


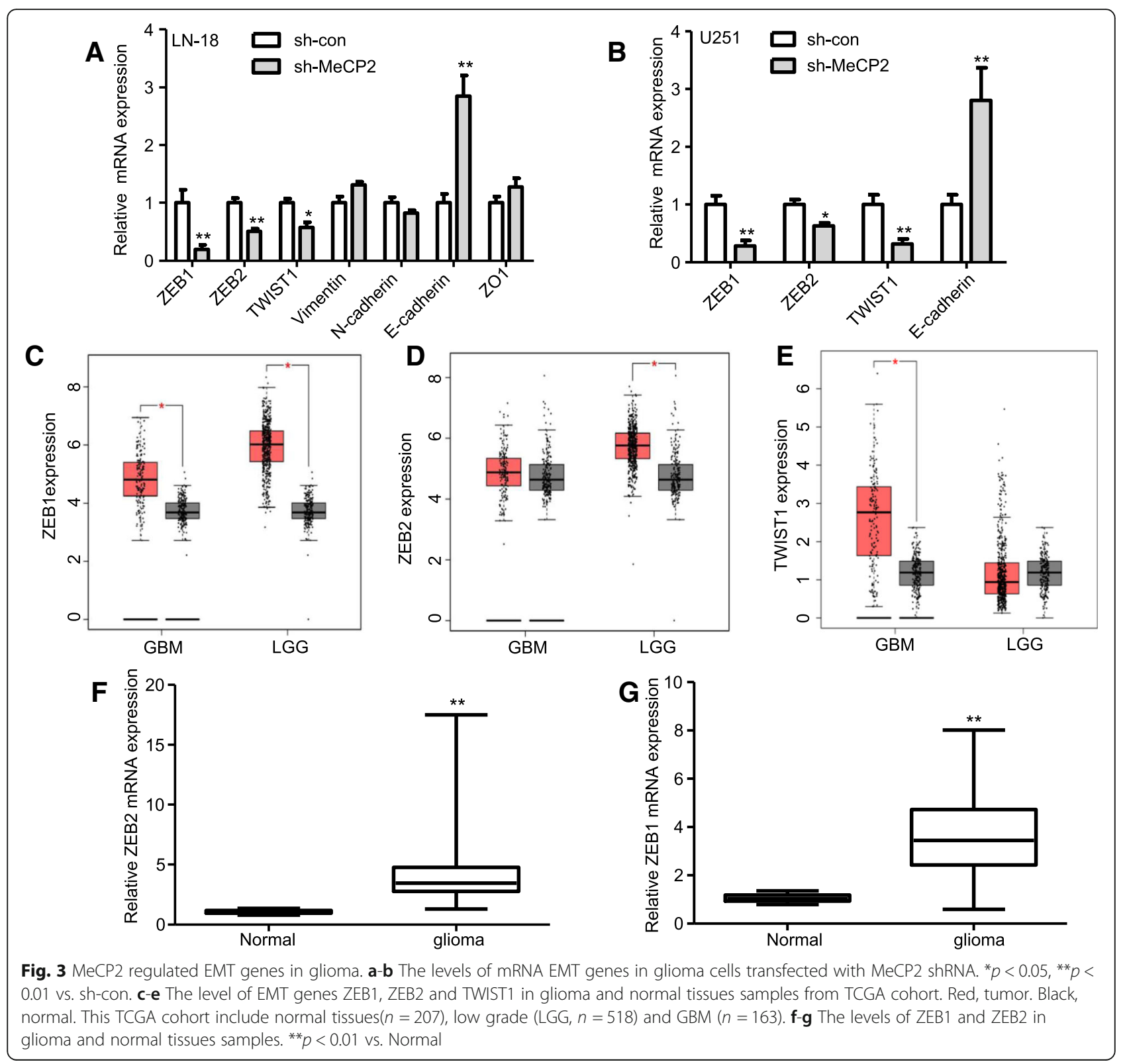

E-Cadherin (Additional file 3: Fig. S1). When measured by western blotting, the expression of ZEB1 and ZEB2 protein was reduced in both LN18 and U251 glioma cells with knockdown of MeCP2 (Fig. 4c-f). All these results indicate a potent role for $\mathrm{MeCP} 2$ in the acquisition of mesenchymal phenotype in gliomas.

\section{MeCP2 knockdown suppresses EMT phenotype in a mouse xenograft model}

To determine the potential impact of MeCP2 expression on EMT in glioma, a xenograft model was used. Sh$\mathrm{MeCP} 2$ resulted in a significant growth reduction compared with sh-con (Fig. 5a). Moreover, ZEB1 and ZEB2 staining were used to detect the EMT in xenografted tumor tissues. As shown in Fig. 5b-c, knockdown of MeCP2 had fewer ZEB1 and ZEB2 positive cells compared with sh-con cells. These data suggested that MeCP2 knockdown in vivo functions similarly as in vitro.

\section{MeCP2 represses miR-200c in glioma}

The miR-200 family can directly target ZEB1 and ZEB2, which served as a pivotal mediator of the EMT process [30]. Therefore, we postulate that MeCP2 may regulate EMT process by miR-200 family. As shown in Fig. 6a-b, expression of miR-200c, but not the other members of miR-200 family, was markedly reduced in MeCP2 


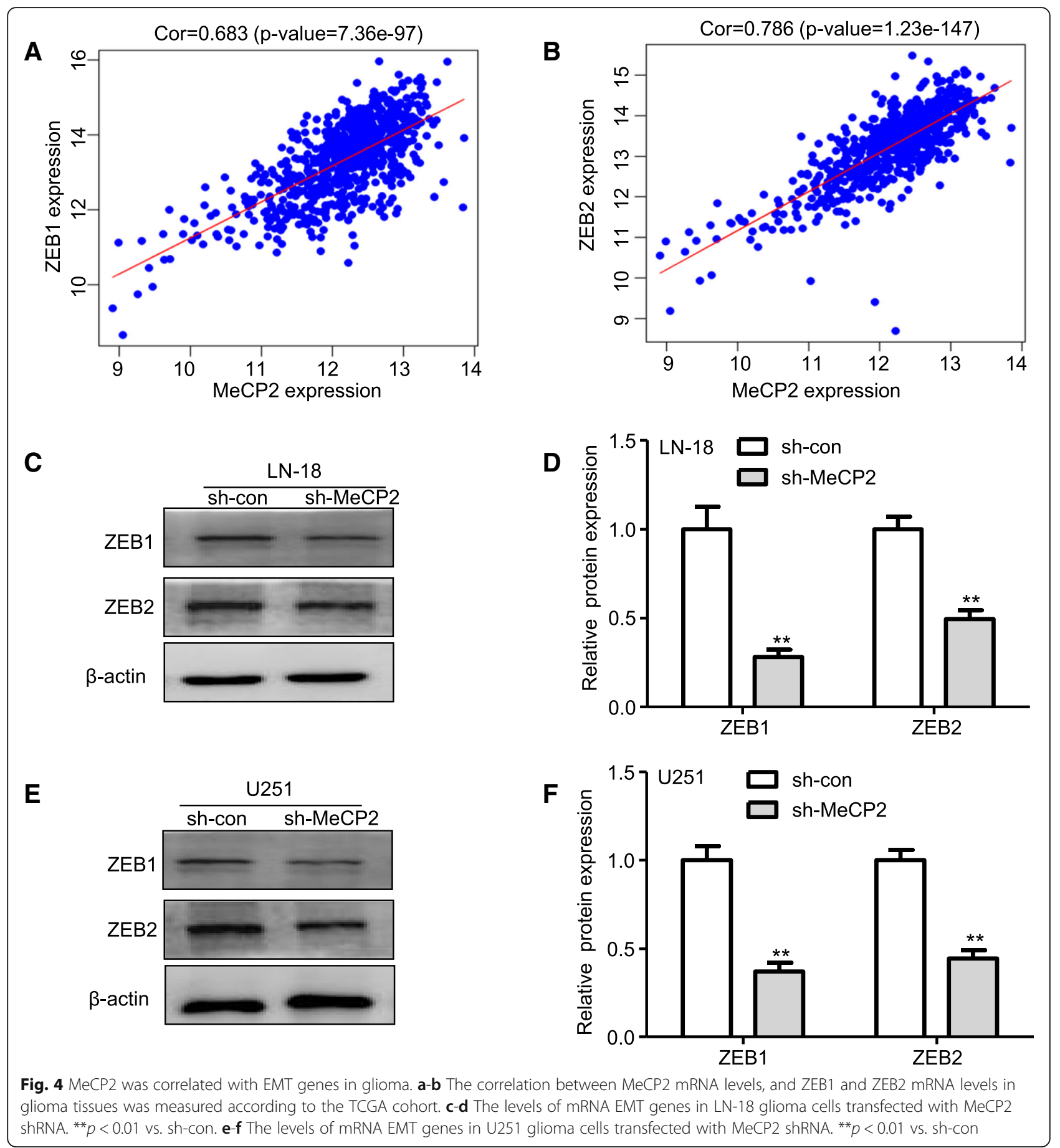

overexpression glioma cells compared with vector. Additionally, MeCP2 knockdown induced miR-200c expression in glioma cells (Additional file 4: Fig. S2a). To investigate whether a relationship exists between MeCP2 expression and miR-200c, miR-200c expression was examined in human glioma samples. The downregulation of miR-200c expression was observed in glioma samples in comparison with normal brain tissues (Fig. 6c). To explore whether expression of miR-200c was related to
$\mathrm{MeCP} 2$, the expression levels of MeCP2 and miR-200c were analyzed in glioma tissues. The correlation of high MeCP2 expression with low miR-200c expression in glioma tissues supports our finding that MeCP2 can downregulate miR-200c in glioma cells (Fig. 6d).

miR-200c is involved in MeCP2 mediated-EMT in glioma To explore whether MeCP2 exerts biological functions via miR-200c, a rescue experiment was performed. As 


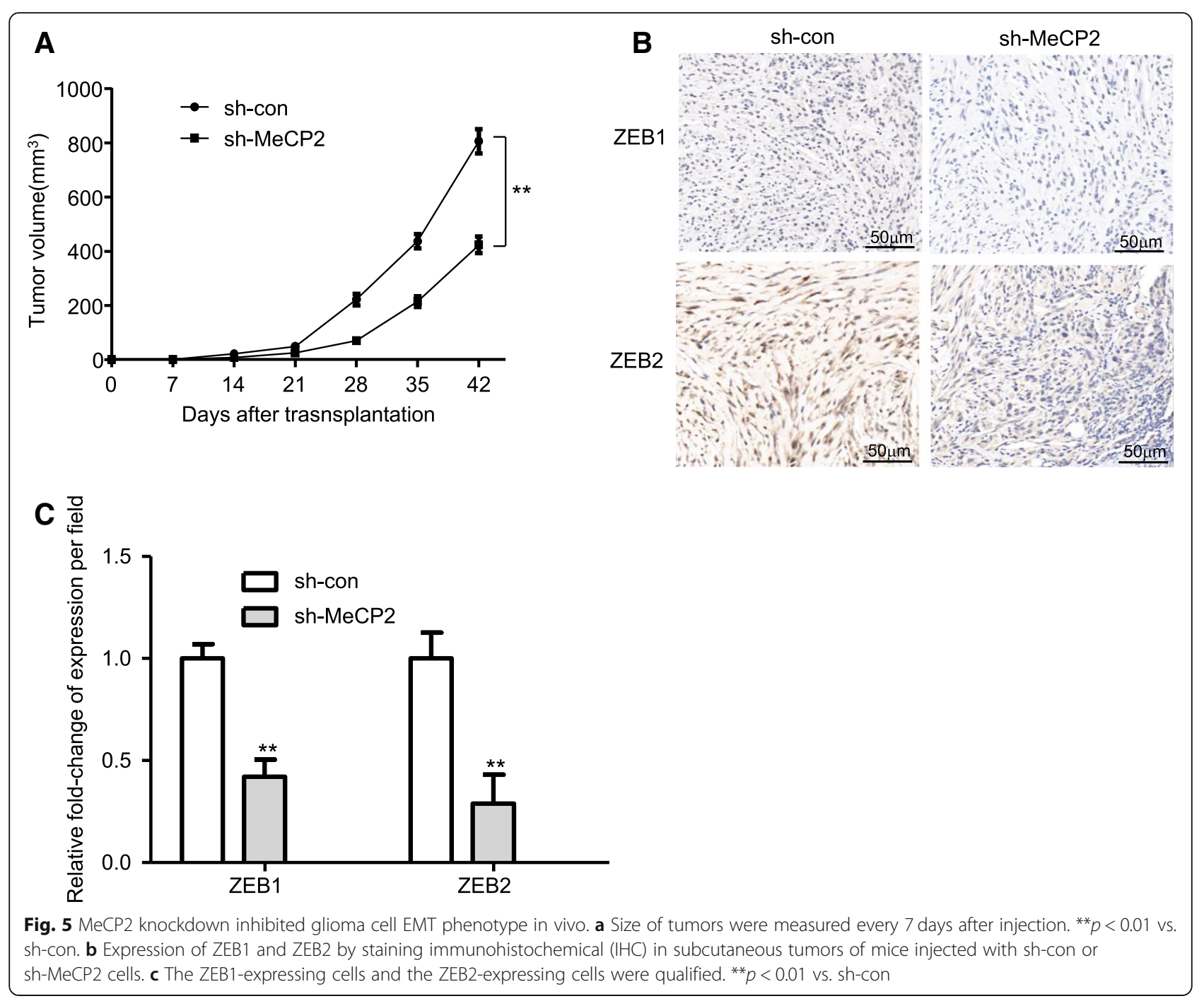

shown in Fig. 7a and Additional file 4: Fig. S2b, MeCP2 overexpression increased spindle shaped morphology, whereas miR-200c markedly reduced spindle shaped morphology induced by MeCP2. Because ZEB1 and ZEB2 is target genes of miR-200c, we wondered whether MeCP2 could modulate ZEB1 and ZEB2 by miR-200c. Ectopic expression of MeCP2 increased ZEB1 and ZEB2 mRNA expression, whereas miR-200c abrogated this increase (Fig. 7b and Additional file 4: Fig. S2c). Additionally, ectopic expression of MeCP2 increased positive ZEB1 and ZEB2 staining, miR-200c abrogated this increase (Fig. 7c and Additional file 4: Fig. S2d). These results may imply that MeCP2 regulate EMT of glioma in part by miR-200c.

\section{MeCP2 as a regulator of miR-200c transcriptional repression}

MeCP2 can mediate transcriptional repression of genes by binding specifically to methylated DNA of genes [31].
To explore the mechanism how MeCP2 repress miR200c expression in a similar manner, we predicted CpG of miR-200c promoter via using MethPrimer. Pyrosequencing results showed that methylation of miR-200c promoter was not significant change in MeCP2 overexpression compared with vector (Additional file 5: Fig. S3a). Previous study showed that $\mathrm{MeCP} 2$ can recruit histone methyltransferase that methylate local H3 lysine 9 (H3K9) are involved in gene silencing [32]. To explore whether MeCP2 represses the level of miR-200c expression via H3K9me3 modification in glioma cells, and the results found that a significant enrichment of H3K9me3 in the miR-200c promoter was observed in MeCP2-overexpressed glioma cells compared with vector cells (Fig. 8a-c). As expected, no alteration in IgG was observed in the miR-200c promoter region (Fig. 8d-e).

To understand how MeCP2 regulates H3K9me3 enrichment in the promoter of miR-200c, we constructed 
A
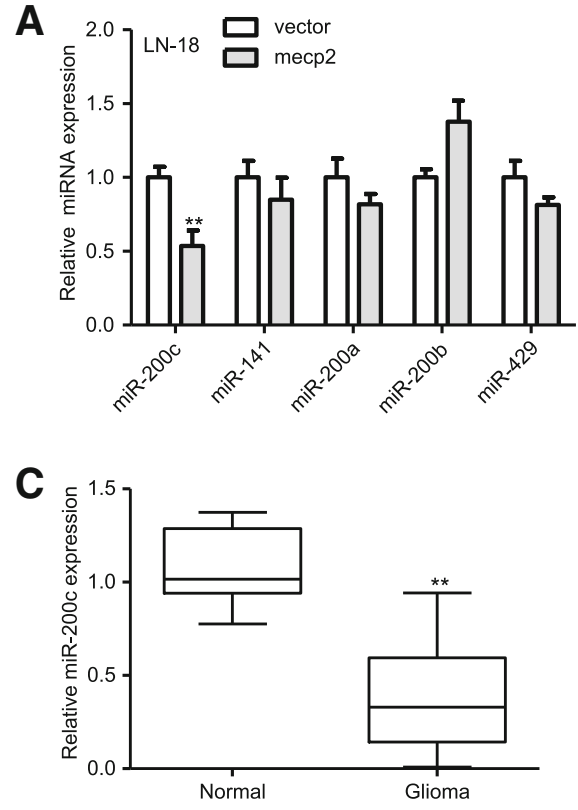

B

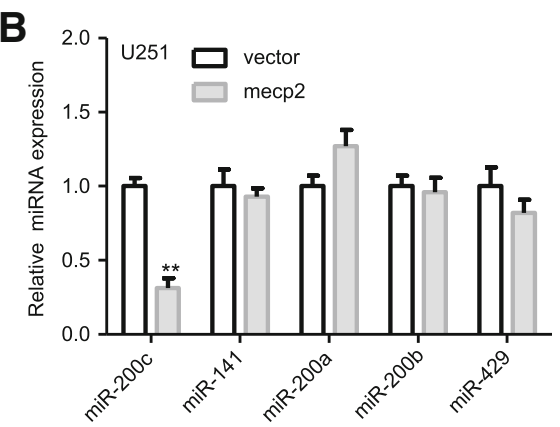

D

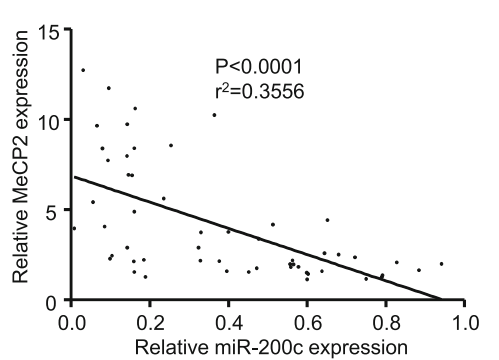

Fig. 6 MeCP2 regulated miR-200 s in glioma. $\mathbf{a}-\mathbf{b}$ The levels of miR-200 s expression in glioma cells transfected with MeCP2 plasmid. **p $<0.01$ vs. vector. $\mathbf{c}$ The levels of miR-200c expression in normal brain and glioma tissues. ${ }^{*} p<0.01$ vs. Normal. $\mathbf{d}$ The correlation between MeCP2 and miR-200c level was measured in glioma tissues

WT, MBD and TRD plasmids to transfect into glioma cells. We found that in lysates of glioma cells, H3K9me3 enrichment in the promoter of miR-200c was significantly increased in MBD and MeCP2 group, but not TRD, compared with vector group (Fig. 8f-g). In addition, we found that miR-200c expression was markedly reduced by expression of either WT or MBD truncated form of MeCP2 (Fig. 8h), suggesting that MBD is capable of exerting transcriptional repression activity as WT MeCP2.

\section{MeCP2 mediated H3K9me3 of miR-200c via its interaction with SUV39H1}

MeCP2 initiates silencing with selective methylation on H3K9, thus creating a high-affinity binding site for suppressor of variegation $39 \mathrm{H} 1$ (SUV39H1) proteins [33].To investigate the interaction between MeCP2 and SUV39H1, coimmunoprecipitation(Co-IP) experiments was used to perform in glioma cells. Endogenous MeCP2 coimmunoprecipitated with endogenous SUV39H1 in glioma cells (Fig. 9a). The interaction of SUV39H1 with $\mathrm{MeCP} 2$ was further confirmed via reverse endogenous coimmunoprecipitation of SUV39H1 with MeCP2 (Fig. 9b), supporting a physical SUV39H1-MeCP2 interaction in vitro. These data prompted us to investigate whether SUV39H1 is involved in H3K9me3 of miR-200c mediated by $\mathrm{MeCP} 2$, ChIP analysis was performed in MeCP2-overexpressing glioma cells. We found that MeCP2 enhanced the binding of SUV39H1 to the promoters of miR-200c (Fig. 9c-d). However, no increase in SUV39H1 binding to the promoters of GAPDH, a gene not mediated by SUV39H1, was observed (Fig. 9c-d). These results indicated that MeCP2 is related to SUV39H1 occupancy at miR-200c promoter. To further confirm whether SUV39H1 mediated epigenetic repression of miR-200c, we examined miR-200c expression after transfection of siSUV39H1 to glioma cells (Additional file 5: Fig. S3b). As shown Fig. 9e-g, SUV39H1 knockdown reduced H3K9me3 occupancy at the promoter of miR-200c and resulted in the increase of miR-200c expression. Collectively, these results revealed that SUV39H1 might be implicated in the function of $\mathrm{MeCP} 2$ as an epigenetic repressor of miR-200c in glioma cells.

\section{Discussion}

Emerging evidences indicate $\mathrm{MeCP} 2$ as a pivotal oncogene in tumorigenesis. MeCP2 as a frequently amplified oncogene has observed in cancer [34-36]. Recently, overexpression of MeCP2 have found in diverse types of cancers, including gastric cancer, endometrial cancers, and prostate cancer, whereas MeCP2 knockdown repressed the proliferation of tumor cells [35, 37, 38]. However, the role of MeCP2 in glioma is still largely unknown. Here, we showed that the expression of MeCP2 was increased in glioma tissues and associated with pathological grade. Interestingly, MeCP2 expression 


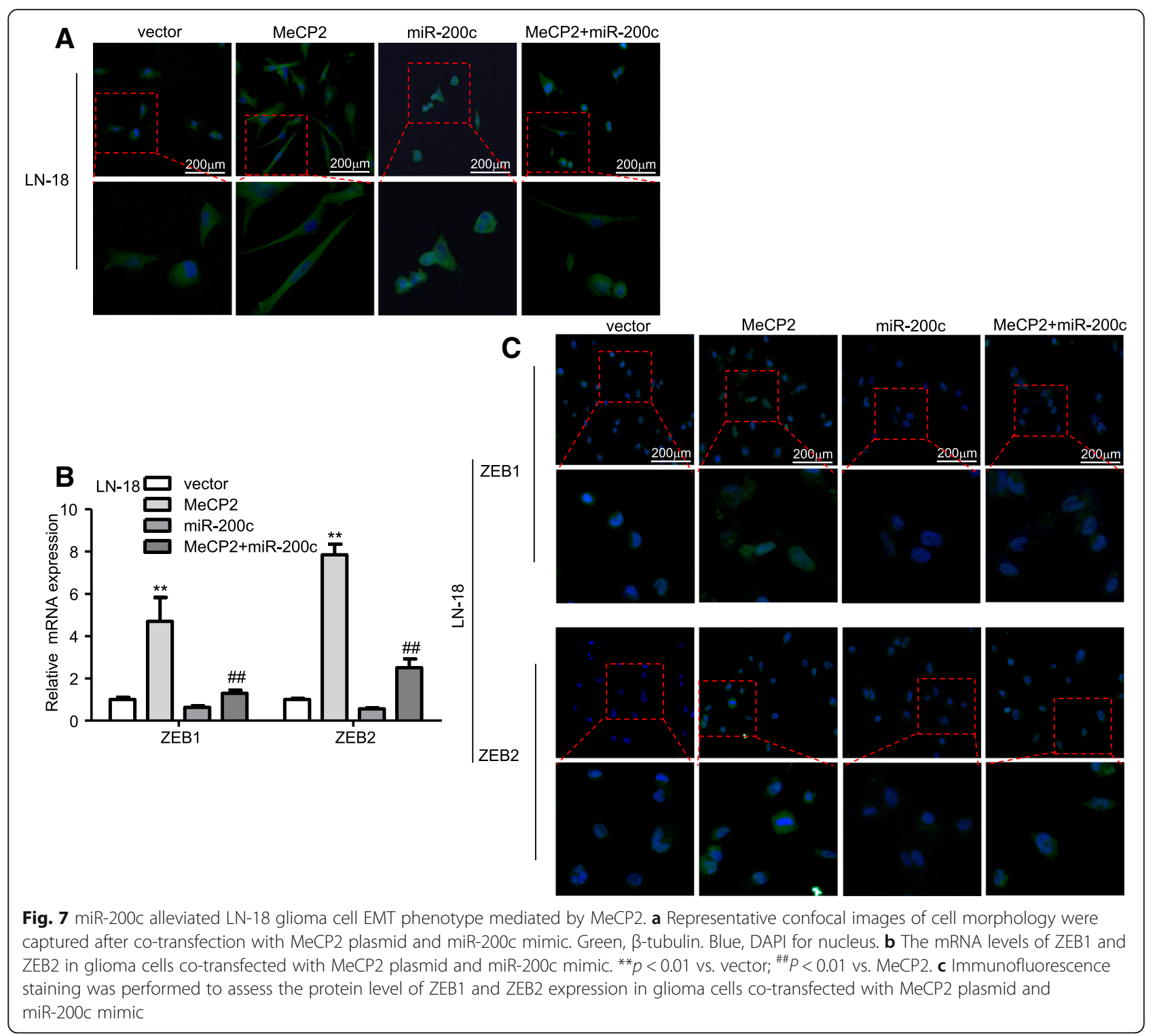

are highly in mesenchymal GBM subtype, suggesting that MeCP2 may be involve in EMT of glioma. Recently, $\mathrm{Li}$ et al. found that MeCP2 promoted EMT by epigenetically silencing BMP7 in endothelial cells [39]. Here, we found that knockdown of MeCP2 significantly induced epithelial phenotype in vitro, whereas overexpression of MeCP2 exerted the opposite effect. These results indicate that MeCP2 may play a key role in the regulation of EMT in gliomas.

EMT is a cellular mechanism that is known to promote a developmental transdifferentiation program [40]. In addition, during EMT, epithelial cells lose their polarity, which will increase express mesenchymal markers, such as ZEB1 and ZEB2, and then leading to acquire invasive potential [41, 42]. This phenomenon has also been related to tumor progression, during which epithelial cells lose signs of differentiation, and obtain enhanced migratory abilities, which results in invasion and metastasis [43, 44]. ZEB1 increases loss of cell-cell contact and therefore fosters increased motility in glioma [45]. Additionally, ZEB2 knockdown inhibited proliferation, migration, invasion, and increased cell death in glioma-derived cell cultures [46]. Here, we found that inhibition of MeCP2 markedly impaired expression of the EMT player ZEB1 and ZEB2 in vitro and in vivo. These results indicated that MeCP2 can function as a transcriptional activator of ZEB1 and ZEB2 in glioma. In this paper, though some important discoveries were disclosed, a subcutaneous tumor model was used in our study, which may result in the 


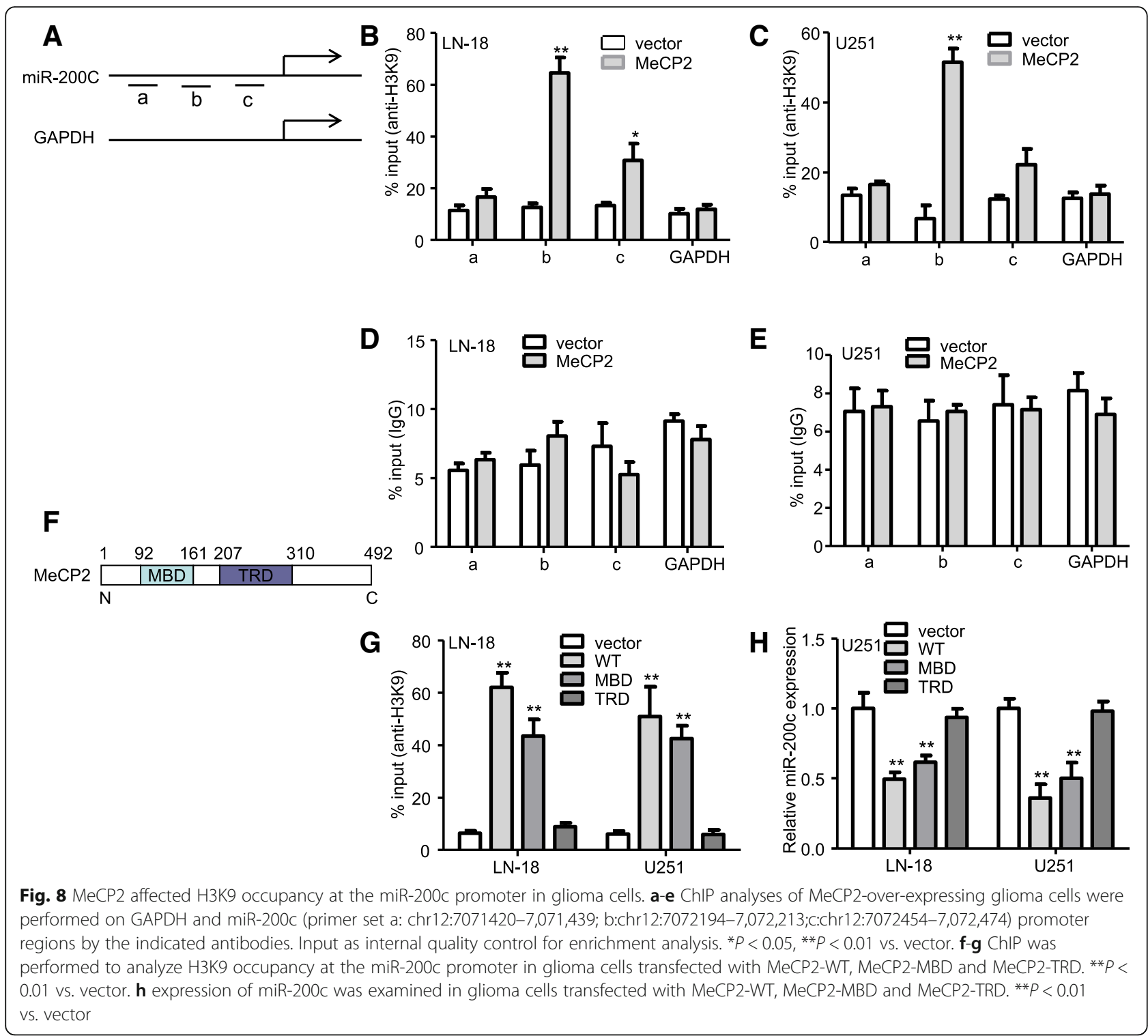

consequences that we may not be able to perfectly simulate the microenvironment of intracranial tumor growth in vivo.

Previous study showed that MeCP2 is best known for its role as a transcriptional repressor in the regulation of gene expression in mammalian cells. MeCP2 was associated with the methylated of TFPI- 2 promoter, and the loss of $\mathrm{MeCP} 2$ in TFPI-2 promoter resulted in gene reactivation in human glioma cells [47]. Recently, it was found that dysregulation of miRNA in cancer is due to the transcriptional repression function of MeCP2 [48, 49]. MiR-200 s family have been reported to play a central role in suppressing EMT marker ZEB1 and ZEB2. Therefore, we speculate that $\mathrm{MeCP} 2$ may mediate a transcriptional activator of ZEB1 and ZEB2 by repressing miR-200s family. Here, we showed that $\mathrm{MeCP} 2$ repressed expression of miR-200c, but not other member of miR-200 family, in glioma cells, and $\mathrm{MeCP} 2$ expression was negatively associated with miR-200c in glioma tissues. In addition, miR200c was involved in MeCP2-mediated EMT in glioma. These results suggest that MeCP2 may regulate EMT, at least in part, by miR-200c in glioma.

$\mathrm{MeCP} 2$ is believed to exert the function of transcriptional inhibition via binding to methylated $\mathrm{CpG}$ dinucleotides [50]. Unexpectedly, we found that overexpression of MeCP2 not changed the methylation of miR-200c promoter. As a master regulator of gene expression, $\mathrm{MeCP} 2$ also acts as a transcriptional repressor by recruiting corepressors and epigenetic regulator, to the promoter regions to suppress a variety of genes 


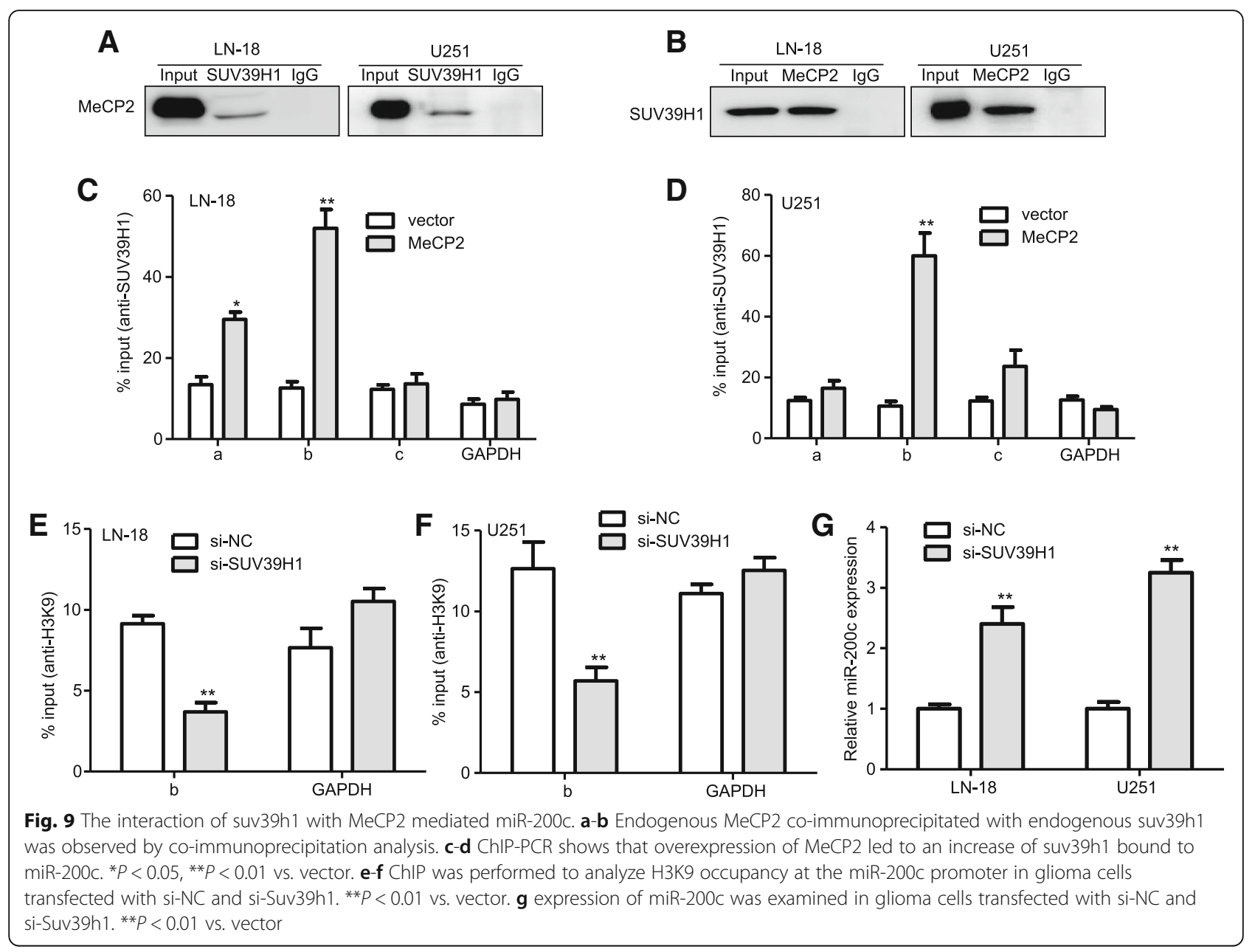

expression [51]. The association of MeCP2 with H3K9 methylation has been reported in the region of IL- 6 gene [32]. A direct correlation between the binding of MeCP2 and the presence of $\mathrm{H} 3 \mathrm{~K} 9 \mathrm{me}$ in promoter of the IкB $\alpha$ gene and the BDNF gene also has been described [52, 53]. Our ChIP results showed, for the first time, that $\mathrm{MeCP} 2$ represses expression of miR-200c by regulating H3K9 methylation of miR-200c promoter. The association of MeCP2 with histone $\mathrm{H} 3$ methyltransferase activity is primarily mediated by methyl-CpG-binding domain (MBD), represents a specific epigenetic mark for transcriptional repression [51].

Here, we showed that MeCP2 mainly exhibit transcriptional repression of miR-200c in glioma depends on its MBD domain, but not TRD domain. As an oncogene, whether MeCP2 exerts its effect in the control of glioma by interacting with other miRNA is still unclear, which is the field we will focus on in subsequent study.

Lunyak et al. reported that MeCP2 initiates silencing with selective methylation on $\mathrm{H} 3 \mathrm{~K} 9$ via interactions with SUV39H1 proteins [33].The molecular mechanism underlying its role in epigenetic regulation is yet unknown. Here, we showed that MeCP2 interacts with SUV39H1 to mediate methylation-based epigenetic transcriptional silencing. Our observation showed that MeCP2 is associated with SUV39H1, and aids in the recruitment of SUV39H1 to the promoter. Then, SUV39H1 recruitment induced in the accumulation of H3K9, which represses miR-200c expression at the transcriptional levels. In consistent with the previous study of MeCP2 interacted with SUV39H1 to regulate their target genes suggests that this pattern of mechanism may be more general.

\section{Conclusions}

In summary, our findings demonstrated that the interaction of MeCP2 with SUV39H1, and deliver H3K9 to the promoter of miR-200c, resulting in the transcriptional repression of the miR-200c, and then activates EMT in glioma (Fig. 10). These results suggest that $\mathrm{MeCP} 2$ as an attractive therapeutic target, the inhibition of which may potentially downregulate the expression of 


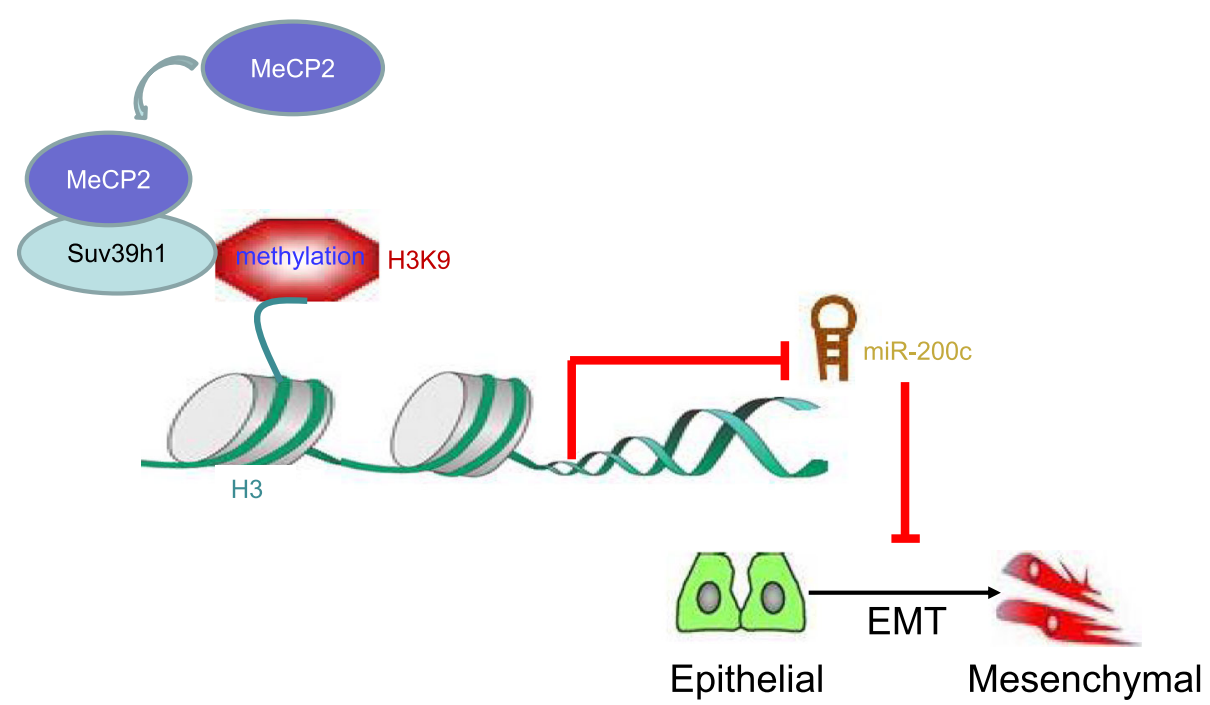

Fig. 10 Schematic diagram showing the mechanism of the interaction of MeCP2 with SUV39H1, and increased the accumulation of the repressive marks H3K9 in the promoter of miR-200c, resulting in the transcriptional repression of the miR-200c, which induced EMT in glioma

miR-200c, resulting in subsequent suppression of the EMT. Hence, our study not only reveals a novel mechanism underlying the epigenetic regulation of EMT in glioma but also provides a novel therapeutic target of glioma. Moreover, identification of MeCP2-specific inhibitor could potentially create a new paradigm in the discovery and development of molecular target therapy for glioma.

\section{Additional files}

Additional file 1: Table S1. The clinicopathological features of glioma patients. (DOC $32 \mathrm{~kb})$

Additional file 2: Table S2. Primers used for GPCR, ChIP, Pyrosequencing Assays. (DOC $214 \mathrm{~kb}$ )

Additional file 3: Figure S1. (A) U251 glioma cell morphology was captured with optical microscope after transfection with MeCP2 shRNA for 2 weeks. (B) The levels of MeCP2 mRNA expression were examined after transfection with MeCP2 plasmid. ${ }^{*} p<0.01$ vs. vector. (C) Representative images of U251 cell morphology were captured after transfection with MeCP2 plasmid. Green, $\beta$-tubulin. Blue, DAPI for nucleus. (D)The level of E-Cadherin in glioma and normal tissues samples from TCGA cohort. Red, tumor. Black, normal. (E-F) The correlation between MeCP2, and E-Cadherin and TWIST1 in glioma tissues was measured according to the TCGA cohort. (DOCX $282 \mathrm{~kb}$ )

Additional file 4: Figure S2. (A) The levels of miR-200c in glioma cells transfected with MeCP2 shRNA. ${ }^{* *} p<0.01$ vs. sh-con. (B) Representative confocal images of U251 glioma cell morphology were captured after co-transfection with MeCP2 plasmid and miR-200c mimic. Green, $\beta$-tubulin. Blue, DAPI for nucleus. (C) The mRNA levels of ZEB1 and ZEB2 in U251 glioma cells co-transfected with MeCP2 plasmid and miR-200c mimic. ${ }^{* *} p<0.01$ vs. vector; ${ }^{\# \#} P<0.01$ vs. MeCP2. (D)

Immunofluorescence staining was performed to assess the protein level of ZEB1 and ZEB2 expression in U251 glioma cells co-transfected with MeCP2 plasmid and miR-200c mimic. (DOCX $279 \mathrm{~kb}$ )
Additional file 5: Figure S3. (A) The methylation of miR-200c promoter was observed after transfection with MeCP2 plasmid. (B) The levels of SUV39H1 mRNA expression were examined after transfection with si-SUV39H1. ** $p<0.01$ vs. sh-con. (DOCX 58 kb)

\section{Abbreviations}

ChIP: Chromatin immunoprecipitation; Co-IP: Co-immunoprecipitation; EMT: Epithelial-to-mesenchymal transition; HDAC: Histone deacetylase complex; MBD: Methyl-CpG-binding domain; MECP2: Methyl CpG-binding protein 2; miRNAs: MicroRNAs; NHA: Normal human astrocytes; RTT: Rett syndrome; SUV39H1: Suppressor of variegation 39H1; TRD: Transcriptional repression domain

\section{Acknowledgements}

Not applicable.

\section{Authors' contributions}

$E B, B Z$ and ZF conceived the project and designed the research. $E B, Y X, X J$, $M C, H W$ performed experiments. EB, XC, ZF analyzed and interpreted the data. EB, XC wrote the manuscript. The study supervisor is BZ, ZF. All authors read and approved the final manuscript.

\section{Funding}

This project was supported by the National Natural Science Foundation of China (No.81402078), Natural Science Foundation of Anhui Province (No.1608085MH225), Key Research and Development Plan Project of Anhui Province (No.1804 h08020270), College Excellent Youth Talent Support Program in Anhui Province (No. gxypZD2019019), Academic Funding Project for Top Talents in Colleges and Universities in Anhui Province (No. gxbjZD10), Nova Pew Plan of the Second Affiliated Hospital of Anhui Medical University (No.2017KA01).

\section{Availability of data and materials}

Additional data are available as Supplementary information.

\section{Ethics approval and consent to participate}

All patients consented to an institutional review board-approved protocol that allows comprehensive analysis of tumor samples (Ethics committee of the Second Affiliated Hospital of Anhui Medical University). This study conforms to the Declaration of Helsinki. 


\section{Consent for publication}

Not applicable.

\section{Competing interests}

The authors declare that they have no competing interests.

\section{Author details}

'Department of Neurosurgery, The Second Affiliated Hospital of Anhui Medical University, 678 Fu Rong Road, Hefei 230601, Anhui Province, China. ${ }^{2}$ Cerebral Vascular Disease Research Center, Anhui Medical University, Hefei 230601, China. ${ }^{3}$ Anhui Province Key Laboratory of Medical Physics and Technology, Center of Medical Physics and Technology, Hefei Institutes of Physical Science, Chinese Academy of Sciences, No. 350, Shushan Hu Road, Hefei 230031, Anhui, China.

Received: 31 May 2019 Accepted: 24 July 2019

Published online: 20 August 2019

\section{References}

1. Huse JT, Holland EC. Targeting brain cancer: advances in the molecular pathology of malignant glioma and medulloblastoma. Nat Rev Cancer. 2010;10(5):319-31.

2. Merchant TE, Li C, Xiong X, Kun LE, Boop FA, Sanford RA. Conformal radiotherapy after surgery for paediatric ependymoma: a prospective study. Lancet Oncol. 2009;10(3):258-66.

3. Verhaak RG, Hoadley KA, Purdom E, Wang V, Qi Y, Wilkerson MD, et al. Integrated genomic analysis identifies clinically relevant subtypes of glioblastoma characterized by abnormalities in PDGFRA, IDH1, EGFR, and NF1. Cancer Cell. 2010;17(1):98-110.

4. Rosmaninho P, Mukusch S, Piscopo V, Teixeira V, Raposo AA, Warta R, et al. Zeb1 potentiates genome-wide gene transcription with Lef1 to promote glioblastoma cell invasion. EMBO J. 2018;37(15).

5. Peng JM, Bera R, Chiou CY, Yu MC, Chen TC, Chen CW, et al. Actin cytoskeleton remodeling drives epithelial-mesenchymal transition for hepatoma invasion and metastasis in mice. Hepatology. 2018;67(6):2226-43.

6. Han SP, Kim JH, Han ME, Sim HE, Kim KS, Yoon S, et al. SNAl1 is involved in the proliferation and migration of glioblastoma cells. Cell Mol Neurobiol. 2011;31(3):489-96.

7. Kahlert UD, Maciaczyk D, Doostkam S, Orr BA, Simons B, Bogiel T, et al Activation of canonical WNT/beta-catenin signaling enhances in vitro motility of glioblastoma cells by activation of ZEB1 and other activators of epithelial-to-mesenchymal transition. Cancer Lett. 2012;325(1):42-53.

8. Picard N, Fagiolini M. MeCP2: an epigenetic regulator of critical periods. Curr Opin Neurobiol. 2019;59:95-101.

9. Adachi M, Monteggia LM. Decoding transcriptional repressor complexes in the adult central nervous system. Neuropharmacology. 2014;80:45-52.

10. Mahgoub M, Adachi M, Suzuki K, Liu X, Kavalali ET, Chahrour MH, et al. MeCP2 and histone deacetylases 1 and 2 in dorsal striatum collectively suppress repetitive behaviors. Nat Neurosci. 2016;19(11):1506-12.

11. Becker A, Zhang P, Allmann L, Meilinger D, Bertulat B, Eck D, et al. Poly(ADPribosyl)ation of methyl $\mathrm{CPG}$ binding domain protein 2 regulates chromatin structure. J Biol Chem. 2016;291(10):4873-81.

12. Wong JJ, Gao D, Nguyen TV, Kwok CT, van Geldermalsen M, Middleton R, et al. Intron retention is regulated by altered MeCP2-mediated splicing factor recruitment. Nat Commun. 2017;8:15134.

13. Chahrour M, Zoghbi HY. The story of Rett syndrome: from clinic to neurobiology. Neuron. 2007;56(3):422-37.

14. Wang YM, Zheng YF, Yang SY, Yang ZM, Zhang LN, He YQ, et al. MicroRNA197 controls ADAM10 expression to mediate MeCP2's role in the differentiation of neuronal progenitors. Cell Death Differ. 2018.

15. Bernard D, Gil J, Dumont P, Rizzo S, Monte D, Quatannens B, et al. The methyl-CpG-binding protein MECP2 is required for prostate cancer cell growth. Oncogene. 2006;25(9):1358-66.

16. Parry L, Clarke AR. The roles of the methyl-CpG binding proteins in cancer. Genes Cancer. 2011;2(6):618-30.

17. Bartel DP. MicroRNAs: genomics, biogenesis, mechanism, and function. Cell. 2004;116(2):281-97.

18. Kiltschewskij D, Cairns MJ. Temporospatial guidance of activity-dependent gene expression by microRNA: mechanisms and functional implications for neural plasticity. Nucleic Acids Res. 2019;47(2):533-45.
19. Treiber T, Treiber N, Meister G. Regulation of microRNA biogenesis and its crosstalk with other cellular pathways. Nat Rev Mol Cell Biol. 2019;20(1):5-20.

20. Tan H, Huang S, Zhang Z, Qian X, Sun P, Zhou X. Pan-cancer analysis on microRNA-associated gene activation. EBioMedicine. 2019;43:82-97.

21. Wong HA, Fatimy RE, Onodera C, Wei Z, Yi M, Mohan A, et al. The Cancer genome atlas analysis predicts MicroRNA for targeting cancer growth and vascularization in glioblastoma. Mol Ther. 2015;23(7):1234-47.

22. Abisoye-Ogunniyan A, Lin H, Ghebremedhin A, Salam AB, Karanam B, Theodore $S$, et al. Transcriptional repressor kaiso promotes epithelial to mesenchymal transition and metastasis in prostate cancer through direct regulation of miR-200c. Cancer Lett. 2018;431:1-10.

23. Feng $X$, Wang Z, Fillmore $R, X i$ Y. MiR-200, a new star miRNA in human cancer. Cancer Lett. 2014;344(2):166-73.

24. Davalos V, Moutinho C, Villanueva A, Boque R, Silva P, Carneiro F, et al. Dynamic epigenetic regulation of the microRNA-200 family mediates epithelial and mesenchymal transitions in human tumorigenesis. Oncogene. 2012:31(16):2062-74.

25. Wiklund ED, Bramsen JB, Hulf T, Dyrskjot L, Ramanathan R, Hansen TB, et al. Coordinated epigenetic repression of the miR-200 family and miR-205 in invasive bladder cancer. Int J Cancer. 2011;128(6):1327-34.

26. Enkhbaatar Z, Terashima M, Oktyabri D, Tange S, Ishimura A, Yano S, et al. KDM5B histone demethylase controls epithelial-mesenchymal transition of cancer cells by regulating the expression of the microRNA-200 family. Cell Cycle. 2013;12(13):2100-12.

27. Tang Z, Li C, Kang B, Gao G, Li C, Zhang Z. GEPIA: a web server for cancer and normal gene expression profiling and interactive analyses. Nucleic Acids Res. 2017;45(W1):W98-W102.

28. Bian EB, Wang YY, Yang Y, Wu BM, Xu T, Meng XM, et al. Hotair facilitates hepatic stellate cells activation and fibrogenesis in the liver. Biochim Biophys Acta Mol basis Dis. 2017;1863(3):674-86.

29. Naito S, von Eschenbach AC, Giavazzi R, Fidler IJ. Growth and metastasis of tumor cells isolated from a human renal cell carcinoma implanted into different organs of nude mice. Cancer Res. 1986;46(8):4109-15.

30. O'Brien SJ, Carter JV, Burton JF, Oxford BG, Schmidt MN, Hallion JC, et al. The role of the miR-200 family in epithelial-mesenchymal transition in colorectal cancer: a systematic review. Int J Cancer. 2018;142(12):2501-11.

31. Gabel HW, Kinde B, Stroud H, Gilbert CS, Harmin DA, Kastan NR, et al. Disruption of DNA-methylation-dependent long gene repression in Rett syndrome. Nature. 2015;522(7554):89-93.

32. Dandrea M, Donadelli $M$, Costanzo C, Scarpa A, Palmieri M. MeCP2/H3meK9 are involved in $\mathrm{L}-6$ gene silencing in pancreatic adenocarcinoma cell lines. Nucleic Acids Res. 2009;37(20):6681-90.

33. Lunyak W, Burgess R, Prefontaine GG, Nelson C, Sze SH, Chenoweth J, et al. Corepressor-dependent silencing of chromosomal regions encoding neuronal genes. Science. 2002;298(5599):1747-52.

34. Liu $Y$, Jin $X$, Li $Y$, Ruan $Y$, Lu Y, Yang $M$, et al. DNA methylation of claudin- 6 promotes breast cancer cell migration and invasion by recruiting MeCP2 and deacetylating $\mathrm{H} 3 \mathrm{AC}$ and $\mathrm{H} 4 \mathrm{AC}$. J Exp Clin Cancer Res. 2016;35(1):120.

35. Tada Y, Brena RM, Hackanson B, Morrison C, Otterson GA, Plass C. Epigenetic modulation of tumor suppressor CCAAT/enhancer binding protein alpha activity in lung cancer. J Natl Cancer Inst. 2006;98(6):396-406.

36. Neupane M, Clark AP, Landini S, Birkbak NJ, Eklund AC, Lim E, et al. MECP2 is a frequently amplified oncogene with a novel epigenetic mechanism that mimics the role of activated RAS in malignancy. Cancer Discov. 2016;6(1):45-58.

37. Chu Y, Wang Y, Zhang G, Chen H, Dowdy SC, Xiong Y, et al. Chromatin composition alterations and the critical role of MeCP2 for epigenetic silencing of progesterone receptor-B gene in endometrial cancers. Cell Mol Life Sci. 2014;71(17):3393-408.

38. Ramachandran K, Gopisetty G, Gordian E, Navarro L, Hader C, Reis IM, et al. Methylation-mediated repression of GADD45alpha in prostate cancer and its role as a potential therapeutic target. Cancer Res. 2009; 69(4):1527-35.

39. Li Z, Song S, Zha S, Wang C, Chen S, Wang F. MeCP2 promotes endothelialto-mesenchymal transition in human endothelial cells by downregulating BMP7 expression. Exp Cell Res. 2019;375(1):82-9.

40. Hanahan D, Weinberg RA. Hallmarks of cancer: the next generation. Cell. 2011;144(5):646-74.

41. De Craene B, Berx G. Regulatory networks defining EMT during cancer initiation and progression. Nat Rev Cancer. 2013;13(2):97-110. 
42. Zheng $H$, Shen $M$, Zha YL, Li W, Wei Y, Blanco MA, et al. PKD1 phosphorylation-dependent degradation of SNAIL by SCF-FBXO11 regulates epithelial-mesenchymal transition and metastasis. Cancer Cell. 2014;26(3):358-73.

43. Shao M, Cao L, Shen C, Satpathy M, Chelladurai B, Bigsby RM, et al. Epithelial-to-mesenchymal transition and ovarian tumor progression induced by tissue transglutaminase. Cancer Res. 2009;69(24):9192-201.

44. Prieto-Garcia E, Diaz-Garcia CV, Garcia-Ruiz I, Agullo-Ortuno MT. Epithelial-tomesenchymal transition in tumor progression. Med Oncol. 2017;34(7):122.

45. Siebzehnrubl FA, Silver DJ, Tugertimur B, Deleyrolle LP, Siebzehnrubl D, Sarkisian MR, et al. The ZEB1 pathway links glioblastoma initiation, invasion and chemoresistance. EMBO Mol Med. 2013;5(8):1196-212.

46. Depner C, Zum Buttel H, Bogurcu N, Cuesta AM, Aburto MR, Seidel S, et al. EphrinB2 repression through ZEB2 mediates tumour invasion and antiangiogenic resistance. Nat Commun. 2016;7:12329.

47. Konduri SD, Srivenugopal KS, Yanamandra N, Dinh DH, Olivero WC, Gujrati $\mathrm{M}$, et al. Promoter methylation and silencing of the tissue factor pathway inhibitor-2 (TFPI-2), a gene encoding an inhibitor of matrix metalloproteinases in human glioma cells. Oncogene. 2003;22(29):4509-16.

48. Dong J, Xiao D, Zhao Z, Ren P, Li C, Hu Y, et al. Epigenetic silencing of microRNA-137 enhances ASCT2 expression and tumor glutamine metabolism. Oncogenesis. 2017;6(7):e356.

49. Cheng TL, Wang Z, Liao Q, Zhu Y, Zhou WH, Xu W, et al. MeCP2 suppresses nuclear microRNA processing and dendritic growth by regulating the DGCR8/Drosha complex. Dev Cell. 2014;28(5):547-60.

50. Chahrour M, Jung SY, Shaw C, Zhou X, Wong ST, Qin J, et al. MeCP2, a key contributor to neurological disease, activates and represses transcription. Science. 2008;320(5880):1224-9.

51. Fuks F, Hurd PJ, Wolf D, Nan X, Bird AP, Kouzarides T. The methyl-CpGbinding protein MeCP2 links DNA methylation to histone methylation. J Biol Chem. 2003;278(6):4035-40.

52. Tian F, Hu XZ, Wu X, Jiang H, Pan H, Marini AM, et al. Dynamic chromatin remodeling events in hippocampal neurons are associated with NMDA receptor-mediated activation of Bdnf gene promoter 1. J Neurochem. 2009; 109(5):1375-88

53. Mann J, Oakley F, Akiboye F, Elsharkawy A, Thorne AW, Mann DA. Regulation of myofibroblast transdifferentiation by DNA methylation and MeCP2: implications for wound healing and fibrogenesis. Cell Death Differ. 2007;14(2): 275-85.

\section{Publisher's Note}

Springer Nature remains neutral with regard to jurisdictional claims in published maps and institutional affiliations.

Ready to submit your research? Choose BMC and benefit from:

- fast, convenient online submission

- thorough peer review by experienced researchers in your field

- rapid publication on acceptance

- support for research data, including large and complex data types

- gold Open Access which fosters wider collaboration and increased citations

- maximum visibility for your research: over $100 \mathrm{M}$ website views per year

At BMC, research is always in progress.

Learn more biomedcentral.com/submissions 